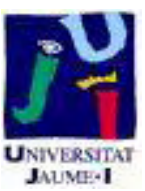

Título artículo / Títol article: Controlled Multibeam Supercontinuum Generation With a Spatial Light Modulator

Autores / Autors

Rocío Borrego Varillas, Jorge Pérez Vizcaíno, Omel Mendoza Yero, Gladys Mínguez-Vega, Javier R. Vázquez de Aldana, Jesús Lancis Sáez

Revista:

Photonics Technology Letters, IEEE, 26(16), 16611664.

Versión / Versió:

Post-print autor

Cita bibliográfica / Cita

BORREGO-VARILLAS, Rocio, et al. Controlled bibliogràfica (ISO 690): Multibeam Supercontinuum Generation With a Spatial Light Modulator. Photonics Technology Letters, IEEE, 2014, vol. 26, no 16, p. 1661-1664.

url Repositori UJI:

http://hdl.handle.net/10234/127345 


\title{
Controlled multi-beam supercontinuum generation with a spatial light modulator
}

\author{
R. Borrego-Varillas, J. Pérez-Vizcaíno, O. Mendoza-Yero, G. Mínguez-Vega, \\ J. R. Vázquez de Aldana, and J. Lancis, Member, IEEE.
}

\begin{abstract}
We report on deterministic femtosecond multifilamentation in fused silica by encoding a diffractive microlens array into a spatial light modulator. The efficiency and focal length of each microlens are modified through the addressing voltage. This allows for a precise control on the energy coupled to the filaments thus obtaining a homogenized supercontinuum pattern from an inhomogeneous irradiance input distribution. Slight changes in the focal length of the microlenses allow for independent tailoring of the supercontinuum spectra.
\end{abstract}

Index Terms-Optical pulse shaping, optical arrays, supercontinuum generation.

\section{INTRODUCTION}

$\mathrm{F}$ ILAMENTATION [1] is a regime of nonlinear propagation where the equilibrium between the self-focusing process, caused by Kerr effect, and the plasma induced defocusing, leads to the self-guiding of light for distances considerably larger than the Rayleigh length [2]. This phenomenon can eventually give rise to an extreme spectral broadening, ranging from the ultraviolet to the infrared regions, termed supercontinuum (SC) generation [3, 4]. Currently, the ongoing research in filamentation ranges from the modeling of the process to the generation of filaments with high flexibility for many different applications.

Spectral control of the SC is desired in applications like femtosecond time-resolved spectroscopy [5], spectral interferometry [6], or in optical parametric amplifiers [7].

When the laser power is much higher than the critical power for self-focusing, the beam undergoes a break-up into multiple filaments. It has been demonstrated that multiple filamentation

This research was funded by the Generalitat Valenciana through project PROMETEO/2012/021 and the Excellence Net about Medical Imaging (project ISIC/2012/013). Financial support from the Pla Propi de Investigació de la Universitat Jaume I through project P1.1B2013-53 is also acknowledged. Authors are also very grateful to the SCIC of the Universitat Jaume I for the use of the femtosecond laser.

R. Borrego-Varillas, J. Pérez-Vizcaíno, O. Mendoza-Yero, G. MínguezVega, and J. Lancis, are with the Departament de Ciències Experimentals, Universitat Jaume I, 12080 Castelló, Spain (e-mail: rborrego@uji.es).

J. R. Vázquez de Aldana, is with the Grupo de Investigación de Microprocesado de Materiales con Láser, Universidad de Salamanca, E37008, Spain

Copyright (C) 2014 IEEE. Personal use of this material is permitted. However, permission to use this material for any other purposes must be obtained from the IEEE by sending a request to pubs-permissions@ieee.org.
(MF) originates from transverse modulation instabilities $[8,9]$, thus producing a random distribution of filaments. Gaining control over the spatial filaments distribution is an important task in applications such as filamentation-based tumor treatment [10], a novel technique which has been recently proposed for cancer therapy. In such case, careful management of both the position and the energy of the filaments, is decisive to control the radiological dose and avoid damage of the adjacent healthy tissue. Another example is the inscription of optical waveguides in dielectrics by direct irradiation with femtosecond pulses that requires the control of the weak plasma generated in the bulk [11]. Although these applications would benefit from MF in terms of speed, they demand for flexible setups that allow for a precise control of the parameters governing the process, such as the energy or the numerical aperture.

Up to now, several strategies have been proposed for deterministic MF: amplitude modulation by a periodic mesh $[12,13]$, nonlinear interaction of two overlapping beams [14], Dammann lenses [15] and wavefront shaping [16], among others. In this sense, focusing the beam by a microlens array is an attractive approach. Watanabe et al. were the first to report a SC array by focusing femtosecond laser pulses into a liquid with a microlens array [17]. Cook and co-workers demonstrated the generation of coherent continuum filaments in B270 glass by using an array of diffractive microlenses (DMLs) [18]. More recently, Camino et al. [19] proposed deterministic MF in fused silica by adjusting the diffraction pattern generated by a loosely focusing $2 \mathrm{D}$ periodic lens array. However, it is still necessary to gain further control over the individual filaments, in terms of energy and spectrum, if real industrial, scientific or biomedical applications want to be developed.

Current megapixel spatial light modulators (SLMs) offer the possibility for versatile and fine control of light beams at the micrometric scale. The use of SLMs has benefited a wide range of applications in adaptive optics [20, 21], scattering media [22] or optical microscopy [23]. In this Letter, we report active control of MF in a solid medium by codifying a diffractive microlens array in a phase-only SLM. We demonstrate for the first time, to our knowledge, precise control on the energy coupled to each one of the filaments thus obtaining a homogeneous SC beam. This is achieved by changing independently the efficiency of each microlens. Moreover, the spectrum of the generated SC was tuned by slightly changing the focal length of the lenses, in agreement with previous observations [24]. 


\section{EXPERIMENT SETUP}

A scheme of the experimental setup is shown in Fig. 1. For the experiment we used a Ti:Sapphire femtosecond laser that emits linearly polarized pulses of about $30 \mathrm{fs}$ (full width half maximum in intensity), centered at $\lambda_{0}=800 \mathrm{~nm}$, with $50 \mathrm{~nm}$ of spectral bandwidth and a repetition rate of $1 \mathrm{kHz}$.

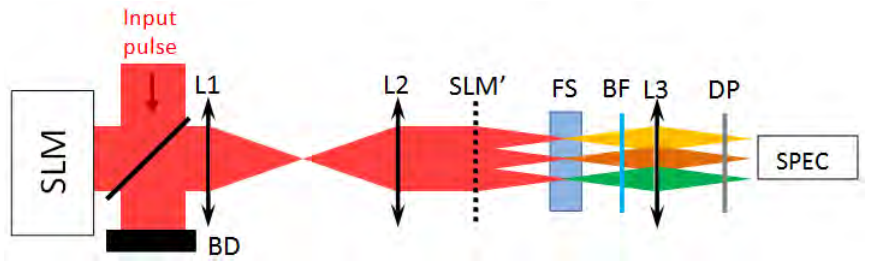

Figure 1. Experimental setup. The input pulse is divided at the 55:45 beam splitter (BD: beam dumper) and reaches the SLM. The pattern generated by the microlens array is focalized in the fused silica (FS) sample. The exiting light is filtered out with a band-pass filter (BF) and collected with a lens and a diffusing plate (DP) into an spectrometer.

The beam was incident on a reflective liquid crystal on silicon SLM (Holoeye Pluto) after reflecting on a beam splitter. The SLM has $1920 \times 1080$ pixels with $8 \mu \mathrm{m}$ of pixel pitch. The reflected beam from the SLM passed through the beam splitter, and then, a $1 \times$ telescope composed by a pair of lenses $\mathrm{L}_{1}$ and $\mathrm{L}_{2}$ of focal lengths $f_{l}=f_{2}=100 \mathrm{~mm}$, imaged the SLM at the focal plane of $\mathrm{L}_{2}$ (labelled as SLM' in Fig. 1).

The phase encoded onto the SLM consisted of an array of $3 \times 3$ lenses $\left(1.73 \times 1.73 \mathrm{~mm}^{2}\right.$ each). The phase for each DML corresponds to that of a kinoform profile given by [15]:

$$
\varphi_{j}(r)=\bmod \left[\frac{\pi r^{2}}{\lambda_{0} f_{j}}, 2 \pi\right]
$$

where $j$-index refers to each DML, $\lambda_{0}$ is the central wavelength of the incident pulse, $f_{j}$ is the focal length, $\mathrm{r}$ is the radial coordinate from the center of the $j$-th lens, and the function $\bmod (x, y)$ gives the remainder on division of $x$ by $y$. This phase ranges from 0 to $2 \pi$. In order to gain control over the energy coupled in this focus for each DMLs Eq. (1) is multiplied by $\alpha_{j}$. The parameter $\alpha_{j}$ varies from 0 to 1 and allows for the control of the diffraction efficiency of the lens $[30,31]$. In this way, the new encoded phase is

$$
\Psi_{j}(r)=\alpha_{j} \varphi_{j}(r)
$$

For $\alpha_{j}=1$ nearly all the light reflected in the corresponding $j$ th lens of the array is directed into the focus. However, when $\alpha_{j} \neq 1$ the energy coupled to the main focus is reduced.

The relation between the value of $\alpha$ and the corresponding efficiency $\eta$ for the generated pattern is given by [25]:

$$
\eta_{j}=\operatorname{sinc}^{2}\left(\alpha_{j}-1\right)
$$

where $\sin c(x)=\sin (\pi x) /(\pi x)$.

The parameters of the codified microlens array were uniformly set to $f_{j}=60 \mathrm{~mm}$ and $\alpha_{j}=1$ in the beginning. A fused silica sample $\left(5 \times 10 \times 20 \mathrm{~mm}^{3}\right.$, all faces polished to optical grade) was placed in such a way that the multiple beams entered through the $5 \mathrm{~mm}$ long face and focused inside the sample (see Fig. 1). The pulse energy, controlled by a variable attenuator, was carefully adjusted just above the threshold to the produce SC with the central beam.
Note that, although sapphire is preferable in terms of stability and bandgap, the energy provided by our laser was not sufficient to generate a 9x9 array due to its larger SC threshold.

At the rear face of the fused silica sample, the nonconverted infrared beam was removed by means of a bandpass filter (FGB37, Thorlabs). The SC light was collected with a lens $\mathrm{L}_{3}\left(f_{3}=100 \mathrm{~mm}\right)$ and projected onto a white screen. Images of the projected SC patterns were recorded with a CMOS camera (Canon EOS, 1100-D). Spectrum measurements of the SC patterns were taken by removing the white screen and placing a diffusing plate and a fiber coupled to a spectrometer (StellarNet Inc, CXR-SR-200) instead. The lateral profiles of the generated filaments were imaged through a $f=60 \mathrm{~mm}$ lens onto a CMOS-based camera (uEye, IDS 1460-C).

\section{EXPERIMENTAL RESULTS}

Owing to the Gaussian distribution of our beam profile the energy is mainly concentrated on the center and, therefore, a different fraction of the incident energy will be delivered to the different microlenses (termed A, B...I, see Fig. 2.a). As a consequence, the values of the efficiency parameters, $\alpha_{j}$, are required to be determined in order to get a uniform distribution of the intensity for all the foci of the microlenses. In order to do that, we measured the threshold pulse energy (incident on the beam-splitter) for SC generation in each one of the 9 microlenses. The threshold is defined as the minimum pulse energy required to just start SC generation (detected with the spectrometer). The measured values ranged from 30 $\mu \mathrm{J}$ for the central microlens (E) to $160 \mu \mathrm{J}$ in the case of one of the external lenses (I). Please note that these values refer to the energy impinging the array, not a single lens. Due to the losses in the beam-splitter and in the SLM, we estimate a $10.5 \%$ of the total energy reaching the fused silica sample. From the threshold energies, the efficiency parameters were individually calculated as the rate

$$
\alpha_{j}=U_{j} / \max \left(U_{j}\right)
$$

and the obtained values were codified in the DLM.

In Fig. 2 b) we show the visible output signal projected on a screen for an input energy of $76 \mu \mathrm{J}$ with all the efficiency parameters set to $\alpha_{j}=1$. As it can be seen in the figure, SC is produced only in the central and the four adjacent foci provided the energy delivered to the external lenses does not exceed the threshold. Moreover, the visible spectrum generated with the 5 central lenses was not identical as expected from the different intensities reached at their corresponding foci. Then, we implemented in the DLM the $\alpha_{j}$ values obtained from Eq. 4 and we increased the input energy to $170 \mu \mathrm{J}$. This value is slightly above the SC threshold to guarantee that no structural damage was inflicted upon the plate. Under this configuration, the energy delivered to all the foci was just above the SC threshold and a homogeneous 
visible signal was generated (see Fig. 2c), both in the spectrum as in the conversion efficiency. An inspection of the fused silica plate revealed that no permanent damage was induced in the sample under these experimental conditions.

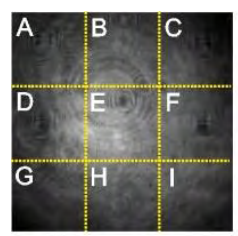

a)

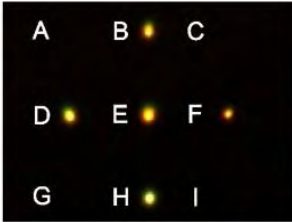

b)

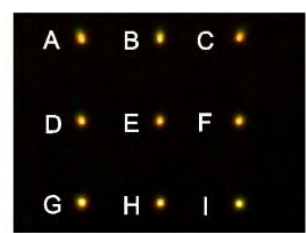

c)
Figure 2. a) Beam profile at the SLM plane for $76 \mu \mathrm{J}$ input energy. The yellow lines denote the edges of the DLMs; b) SC signal for $76 \mu \mathrm{J}$ input energy and c) SC signal for $170 \mu \mathrm{J}$ input energy with efficiency correction as explained in the text.

So far, we have corrected the influence of spatial inhomogeneties of the incident beam in the intensity and spectrum of the SC via the parameters $\alpha_{j}$. In a second set of experiments, we pursued control over the spectral properties of the so-generated beams. A direct way to do that is, for instance, increasing the incident pulse energy. However, in practical applications an increase in the input energy is undesirable because it could easily lead to a permanent damage in the sample [26]. Recently, it has been demonstrated [24] that when a femtosecond beam is focused with a diffractive lens, wavelength tunability in the SC signal can be achieved by simply changing the lens-sample relative distance. It was found that the depth at which the filament is formed is closely connected to the SC spectrum [27]. Based on this, the position of the filament, and thus the spectral content of the SC generated signal, could be controlled by slightly modifying the focal length of the DMLs and keeping the pulse energy constant. Obviously, when changing the focal length, the numerical aperture of the focusing beams is also slightly changed.
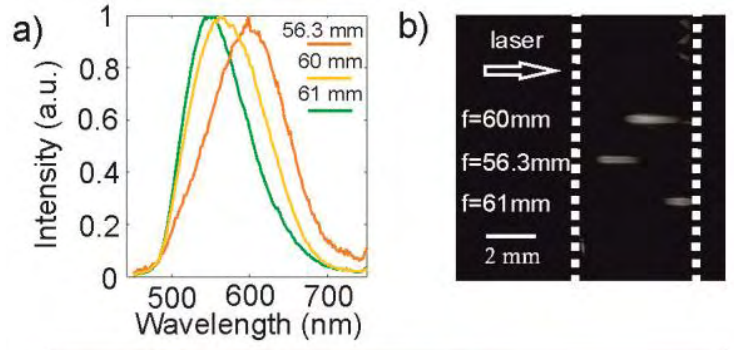

C)

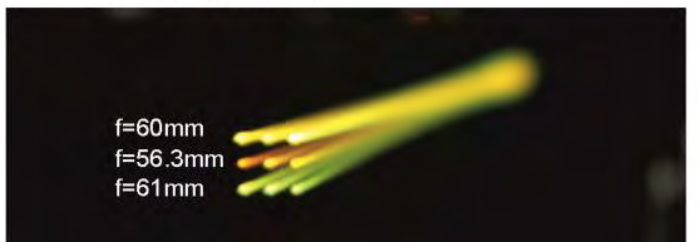

We implemented this idea in the SLM. The pulse energy was set to $200 \mu \mathrm{J}$ and the values of $\alpha_{j}$ were set to the computed values for foci homogeneization. Then, we codified in the DML phases corresponding to different focal lengths. As expected, the spectra of the generated SC changed in agreement with our expectations, as it can be seen in Fig. 3 b. As an example of the flexibility of our setup, we set different focal lengths for each row of lenses (i.e. $f_{A}=f_{B}=f_{C}=61 \mathrm{~mm}$, $\mathrm{f}_{\mathrm{D}}=\mathrm{f}_{\mathrm{E}}=\mathrm{f}_{\mathrm{F}}=56.3 \mathrm{~mm}$ and $\mathrm{f}_{\mathrm{G}}=\mathrm{f}_{\mathrm{H}}=\mathrm{f}_{\mathrm{I}}=60 \mathrm{~mm}$ ). The filaments produced inside the silica sample, moved accordingly (see Fig. 3a). Moreover, the DMLs with the longer focal lengths (A-C) formed short filaments, clipped by the rear face of the sample. This is associated with spectra tuned towards the shortest wavelengths, which is in accordance with the observations in [26]. In contrast, for the shortest focal lengths (D-F), filaments developed slightly behind the front face. Under these conditions, three colored patters were observed at the output of the sample (see Fig. 3c).

\section{CONCLUSION}

In conclusion, we have reported controlled MF in fused silica by encoding a DML array in a phase-only SLM for SC generation. We have demonstrated a precise and independent control on (i) the energy coupled to each of the filaments by means of the $\alpha$ parameter, and (ii) the spectrum of the generated SC light by changing the focal length of the DMLs. We believe that the results of these investigations can be useful in applications which demand for homogenized MF patterns and/or spectral tunability, including femtosecond laser inscription, time-resolved spectroscopy or tumor treatment.

\section{REFERENCES}

[1] A. Braun, G. Korn, X. Liu, D. Du, J. Squier, and G. Mourou, "Selfchanneling of high-peak-power femtosecond laser-pulses in air", Optics Letters 20, 73-75 (1995).

[2] A. Couairon and A. Mysyrowicz, "Femtosecond filamentation in transparent media," Physics Reports-Review Section of Physics Letters 441, 47-189 (2007).

[3] R. R. Alfano and S. L. Saphiro, "Emission in the Region 4000 to $7000 \AA$ Aia Four-Photon Coupling in Glass," Physical Review Letters 24, 584-587 (1970).

[4] R. R. Alfano, The Supercontinuum Laser Source (Springer, 2006).

[5] V. I. Klimov and D. W. McBranch, "Femtosecond high-sensitivity, chirp-free transient absorption spectroscopy using kilohertz lasers," Optics Letters 23, 277-279 (1998).

[6] Z. W. Wilkes, S. Varma, Y. H. Chen, H. M. Milchberg, T. G. Jones, and A. Ting, "Direct measurements of the nonlinear index of refraction of water at 815 and $407 \mathrm{~nm}$ using single-shot supercontinuum spectral interferometry," Applied Physics Letters 94, 211102-211103 (2009).

[7] G. Cerullo and S. De Silvestri, "Ultrafast optical parametric amplifiers," Review of Scientific Instruments 74, 1-18 (2003).

[8] H. Schroeder, J. Liu, and S. L. Chin, "From random to controlled small-scale filamentation in water," Optics Express 12, 4768-4774 (2004).

Figure 3. Filaments' profiles inside the fused silica sample (the dotted lines indicate the sample's input and output faces); b) corresponding spectra measured behind the FGB37 filter and c) photograph of the generated SC light. 
[9] L. Bergé, S. Skupin, F. Lederer, G. Méjean, J. Yu, J. Kasparian, E. Salmon, J. P. Wolf, M. Rodríguez, L. Wöste, R. Bourayou, and R. Sauerbrey, "Multiple filamentation of terawatt laser pulses in air," Physical Review Letters 92, 225002-225005 (2004).

[10] R. Meesat, H. Belmouaddine, J.-F. Allard, C. Tanguay-Renaud, R. Lemay, T. Brastaviceanu, L. Tremblay, B. Paquette, J. R. Wagner, J.-P. Jay-Gerin, M. Lepage, M. A. Huels, and D. Houde, "Cancer radiotherapy based on femtosecond IR laser-beam filamentation yielding ultra-high dose rates and zero entrance dose," Proceedings of the National Academy of Sciences 109, E2508-E2513 (2012).

[11] R. Osellana, G. Cerullo, and R. Ramponi, Femtosecond laser micromachining (Springer Berlin, 2012).

[12] V. P. Kandidov, N. Akozbek, M. Scalora, O. G. Kosareva, A. V. Nyakk, Q. Luo, S. A. Hosseini, and S. L. Chin, "Towards a control of multiple filamentation by spatial regularization of a high-power femtosecond laser pulse," Applied Physics B 80, 267-275 (2005).

[13] O. G. Kosareva, T. Nguyen, N. A. Panov, W. Liu, A. Saliminia, V. P. Kandidov, N. Akozbek, M. Scalora, R. Vallee, and S. L. Chin, "Array of femtosecond plasma channels in fused silica," Optics Communications 267, 511-523 (2006).

[14] J. Liu, H. Schroeder, S. L. Chin, R. Li, and Z. Xu, "Ultrafast control of multiple filamentation by ultrafast laser pulses," Applied Physics Letters 87, 161105 (2005).

[15] J. Pérez-Vizcaíno, O. Mendoza-Yero, R. Borrego-Varillas, G. Mínguez-Vega, J. R. Vázquez de Aldana, and J. Lancis, "On-axis non-linear effects with programmable Dammann lenses under femtosecond illumination," Optics Letters 38, 1621-1623 (2013).

[16] C. P. Hauri, J. Biegert, U. Keller, B. Schaefer, K. Mann, and G. Marowski, "Validity of wave-front reconstruction and propagation of ultrabroadband pulses measured with a Hartmann-Shack sensor," Optics Letters 30, 1563-1565 (2005).

[17] W. Watanabe, Y. Masudda, H. Arimoto, and K. Itoh, "Coherent array of white-light continuum generated by microlens array," Optical Review 6, 167-172 (1999).

[18] K. Cook, R. McGeorge, A. K. Kar, M. R. Taghizadeh, and R. A. Lamb, "Coherent array of white-light continuum filaments produced by diffractive microlenses," Applied Physics Letters 86, 021105 (2005).
A. Camino, Z. Hao, X. Liu, and J. Lin, "Control of laser filamentation in fused silica by a periodic microlens array," Opt. Express 21, 7908-7915 (2013).

[20] J. Arines, V. Durán, Z. Jaroszewicz, J. Ares, E. Tajahuerce, P. Prado, J. Lancis, S. Bara, and V. Climent, "Measurement and compensation of optical aberrations using a single spatial light modulator," Optics Express 15, 15287-15292 (2007).

[21] S. Ngcobo, I. Litvin, L. Burger and A. Forbes, "A digital laser for on-demand laser modes," Nature Communications 4, 2289 (2013).

[22] I. M. Vellekoop and A. P. Mosk, "Focusing coherent light through opaque strongly scattering media," Optics Letters 32, 2309-2311 (2007).

[23] C. Maurer, A. Jesacher, S. Bernet, and M. Ritsch-Marte, "What spatial light modulators can do for optical microscopy," Laser Photonics Review 5, 81-101 (2011).

[24] C. Romero, R. Borrego-Varillas, A. Camino, G. Minguez-Vega, O. Mendoza-Yero, J. Hernandez-Toro, and J. R. Vázquez de Aldana, "Diffractive optics for spectral control of the supercontinuum generated in sapphire with femtosecond pulses," Optics Express 19, 4977-4984 (2011).

[25] V. Moreno, J. F. Roman, and J. R. Salgueiro, "High efficiency diffractive lenses: Deduction of kinoform profile," American Journal of Physics 65, 556-562 (1997).

[26] C. B. Schaffer, A. Brodeur and E. Mazur, "Laser-induced breakdown and damage in bulk transparent materials induced by tightly focused femtosecond laser pulses," Meas. Sci. Technol. 12, 1784-1794 (2001).

[27] R. Borrego-Varillas, C. Romero, O. Mendoza-Yero, G. MínguezVega, I. Gallardo, and J. R. Vázquez de Aldana, "Femtosecond filamentation in sapphire with diffractive lenses," J. Opt. Soc. Am. B 30, 2059-2065 (2013) 